\title{
Adverse Impact of Rheumatoid Arthritis on Pregnancy Outcomes: A Saudi Arabia Prospective Multicenter Study
}

\author{
Hanan Al Rayes $\mathbb{D D}^{\prime}$ \\ Sultana Abdulaziz ${ }^{2}$ \\ Asma Mohammed Alotaibi ${ }^{3}$ \\ Mufaddal Adil Alaithan (D) ${ }^{\prime}$ \\ Mohammed Attar ${ }^{4}$ \\ Hassan Daghasi ${ }^{4}$ \\ Rand Melibari ${ }^{2}$ \\ Ali $\mathrm{H}$ Althagafi ${ }^{4}$ \\ Basant Elnady (1D) 4,5
}

'Rheumatology Division, Department of Medicine, Prince Sultan Military Medical City, Riyadh, Saudi Arabia; ${ }^{2}$ Division of Rheumatology, Department of Medicine, King Fahad Hospital, Jeddah, Saudi Arabia; ${ }^{3}$ Department of Medicine, Prince Sultan Military Medical City, Riyadh, Saudi Arabia; ${ }^{4}$ Division of Rheumatology, Department of Medicine, Al Hada Armed Forces Hospital, Taif, Saudi Arabia; ${ }^{5}$ Rheumatology, Rehabilitation and Physical Medicine Department, Faculty of Medicine, Benha University, Benha, Egypt

Correspondence: Basant Elnady Division of Rheumatology, Department of Medicine, Al Hada Armed Forces Hospital, Al Hada Ring Road, Al Hada, Postal Code 1347, Taif, Zip Code 21944, Saudi Arabia

Tel +966562387723

Fax +966I275428II

Email basantelnady@gmail.com
Background: Rheumatoid arthritis (RA) is associated with adverse pregnancy outcomes and postpartum complications, especially with severe disease activity.

Objective: The current study aimed to compare the pregnancy outcomes in patients with RA and healthy controls as well as to assess the impact of disease-related variables, medications and disease activity on pregnancy and neonatal-related outcomes in the Saudi population.

Methods: This prospective multicenter study included pregnant female patients with RA from three tertiary centers in Saudi Arabia. The demographics, disease activity scoring (DAS28-CRP), medication before and during pregnancy, pregnancy-related outcomes, and complications in comparison to age-matched healthy female controls were noted.

Results: A total of 77 pregnant patients with RA and 250 healthy age-matched pregnant controls were included in the study. A total of $67.53 \%$ were in remission before conception (DAS28CRP $\leq 2.6$ ), and $81.8 \%$ of pregnancies were planned. Age of conception, preterm labor, neonatal intensive care unit (NICU) admission and low birth weight were statistically significant $(\mathrm{p}<0.05)$ and higher in RA patients than in healthy controls. Longer disease duration $(\mathrm{p}<0.001)$, and high $\mathrm{C}$-reactive protein and erythrocyte sedimentation rate levels before conception ( $\mathrm{p} \leq 0.001$ ) were statistically associated with preterm NICU admission. There was statistically significant association between mild $(\mathrm{p}=0.015)$ or moderate to severe DAS28-CRP ( $p=0.001)$ and RA patients regarding pregnancy outcomes. The classification table obtained from the logistic model showed patients with mild and moderate-severe DAS28-CRP have significantly high chances of having an adverse pregnancy outcome.

Conclusion: RA has a negative impact on pregnancy-related outcomes. Higher disease activity is considered a major risk; thus, tight disease control should be aimed. Planned pregnancy follow-up is associated with better pregnancy outcomes.

Keywords: rheumatoid arthritis, pregnancy, disease activity

\section{Key Points}

1. This study proposed, for the first time, to medical records review with follow-up to prospectively study pregnancy outcomes in Saudi patients with RA from three large tertiary hospitals.

2. RA patients should plan their pregnancies in terms of pregnancy timing, therapy modifications, and prenatal and postnatal follow-ups to reduce undesirable outcomes.

3. Despite the optimal management of disease activity during pregnancy, RA patients in remission harbor the risk of preterm labor with low birth weight and frequent neonatal newborn intensive care unit admission. 


\section{Introduction}

Rheumatoid arthritis (RA) is a common autoimmune disease that predominantly affects the skeletal system and connective tissues. RA usually targets women of childbearing age. ${ }^{1}$ Women might avoid getting pregnant for fear of any adverse effect on their pregnancy such as the newborn might inherit the disease or medications for RA might negatively impact pregnancy outcome. ${ }^{2}$

It has long been known that RA symptoms might improve during pregnancy with variable percentages, but only a few studies used an objective disease activity score of 28-joint count $\mathrm{C}$ reactive protein (DAS28-CRP) to prove this assumption. ${ }^{3}$ The relationship of disease control in Caucasian RA patients during pregnancy has a favorable outcome. ${ }^{4}$ However, high disease severity is frequently associated with adverse pregnancy outcomes, such as size of the gestational newborns, preterm labor, increased number of cesarean sections, and pre-eclampsia with a controversial result. ${ }^{1,2,5}$

Recent data suggest that infants born small for gestational age and/or prematurely carry a higher risk for shortand long-term complications, ranging from higher rates of neonatal intensive care unit (NICU) admissions to adulthood morbidities. ${ }^{6,7}$ The impact of RA on pregnancy and vice versa has not yet been extensively highlighted in different ethnicities such as the Saudi population. The current study aimed to assess the impact of RA on pregnancy and neonatal outcomes in the Saudi population in comparison to healthy controls and to study the effect of disease-related variables, medications and disease activity on pregnancy and neonatal-related outcomes.

\section{Materials and Methods Study Design}

The present study was conducted at three tertiary centers in Saudi Arabia, Prince Sultan Military Medical City (PSMMC) in Riyadh, King Fahad Hospital in Jeddah, and Al-Hada Armed Forces Hospital in Taif. This was a multicenter case-control prospective study in the Saudi population. The primary outcome was to assess the effect of RA on pregnancy, primarily the obstetric complications and fetal outcomes, in comparison to healthy age-matched pregnant women. The secondary outcome was to assess the impact of disease-related clinical and laboratory variable, medications and disease activity (using DAS28-CRP) on pregnancy outcomes.

\section{Study Population}

Patients with RA who were following up in the rheumatology clinics from January 2018 to January 2020 were recruited from three tertiary hospitals. Participants aged $>18$ years meeting the following inclusion criteria were enrolled: patients should be pregnant with RA before the gestational week of 12, fulfilling the ACR/EULAR 2010 criteria for $\mathrm{RA}^{8}{ }^{8}$ on regular follow-up visits at least every 2 months in rheumatology clinic, with at least one measured DAS28CRP done by Rheumatologist periconceptional, first, second and third trimester during follow-up, on medication with conventional synthetic disease modifying antirheumatic drugs (csDMARDs) and/or biological DMARDs (bDMARDs) or targeted synthetic DMARDs (tsDMARDs), and following up at Rheumatology clinic in the three tertiary hospitals. The inclusion criterion for the controls was agematched healthy pregnant women without comorbidities.

The study exclusion criteria were any major comorbidity known to affect the pregnancy outcome in both groups, such as overlap with other rheumatic diseases, diabetes mellitus, hypertension, chronic renal disease, ovarian surgery, uterine anomalies, prior chemotherapeutic treatment of ectopic pregnancy, prior exposure to known or possible ovary-toxic medications, and twin pregnancy.

\section{Methods}

Participants in both groups underwent full history taking including clinical and laboratory assessment via direct interview during regular follow-up at rheumatology clinic for RA patients and prenatal care clinics for healthy controls. Data were collected in the research room or the clinic at the time of routine follow-up with the help of a research assistant, to report if the pregnancy was planned by both patient and rheumatologist with medication modification, or occurred without a planned protocol, patient physician protocol defined as prior disease activity control and medication adjustment before conception.

Laboratory variables included measurements of levels of Rheumatoid factor (RF), anti-cyclic citrullinated peptide antibodies (anti-CCP Abs) using the chemiluminescence microparticle immunoassay (CMIA), erythrocyte sedimentation rate ESR using the Westergren method, and CRP using the enzyme-linked immunosorbent assay (ELISA) technique.

Pregnancy outcomes in both groups were reported, including obstetric complications preeclampsia, gestational diabetes, preterm birth which defined as Preterm or premature birth refers to a delivery that occurs between 20 and 37 
weeks of gestation, ${ }^{9}$ elective termination of pregnancy. Fetal outcomes included Low birth weight (LBW) Less than 2500 grams, ${ }^{10}$ congenital anomalies, NICU admission, stillbirth, live birth. For each live birth, the age of gestation was assessed, and any reported congenital anomalies were noted. Disease activity was assessed using the DAS28-CRP score $^{11}$ and recorded before and during pregnancy at the follow-up visits every trimester. Medications including antiinflammatory or immunosuppressant drugs before and during pregnancy were also documented.

\section{Ethical Approvals}

This study complied with the Declaration of Helsinki and was approved by the local ethics research committees of Prince Sultan Military Medical City (PSMMC) in Riyadh (HP-01R079), Al-Hada Armed Forces Hospital (H-02-T-078), and King Fahad Hospital in Jeddah (H-02-J-002). Written informed consent was obtained from all the patients and controls.

\section{Statistical Analysis}

Data were entered and analyzed using IBM SPSS statistics software for Windows, version 26.0 (Armonk, NY: IBM Corp). The data were represented as the mean and standard error of the mean (SEM) for quantitative variables. Frequencies and percentages were reported for the qualitative variables. The Welch $t$-test was used to observe the mean differences between the two groups. Welch one-way ANOVA was applied to compare the mean differences between more than two groups. Pearson chi-square and Fisher's exact tests were used to find associations between the qualitative variables (various parameters between pregnancy cases in RA and pregnancy healthy controls and RA pregnancy-related outcomes with smoking, medications used during pregnancy and disease activity scoring at conception). Statistical significance was set at $\mathrm{p}<0.05$. Binary logistic regression analysis. The categorical dependent variable was adverse pregnancy outcome (Yes, No). Value of model chi-square and HosmerLemeshow was significant at 5\% level of significance confirming that the fitted model was appropriate.

\section{Results}

A total of 77 pregnant women with RA and 250 healthy pregnant controls were recruited for the study. The mean age of the participants at conception was $32.78 \pm 0.69$ years for RA patients and $30.32 \pm 0.84$ for controls, for RA patient's disease duration was $9.16 \pm 0.55$ years. The majority $(67.53 \%)$ of the patients were in remission before conception (DAS28-CRP $\leq 2.6$ ), and $81.8 \%$ of pregnancies were planned by both patients and the consulting rheumatologists with medication modification. The demographic data of the study group are illustrated in Table 1.

Table I Demographics and Clinical Data of Rheumatoid Arthritis Patients

\begin{tabular}{|c|c|}
\hline RA Group $(n=77)$ & Data \\
\hline Age at pregnancy ${ }^{*}$, mean \pm SEM, years & $32.78 \pm 0.69^{*}$ \\
\hline Age of disease onset ${ }^{*}$, mean \pm SEM, years & $26.49 \pm 0.82^{¥}$ \\
\hline Duration of disease ${ }^{¥}$, mean \pm SEM, years & $9.16 \pm 0.55^{*}$ \\
\hline \multicolumn{2}{|l|}{ RF-CCP Status, $n(\%)^{*}$} \\
\hline Seropositive & 47 (61.04\%) \\
\hline Smoking (yes), n (\%) & $5(6.49 \%)$ \\
\hline Planned pregnancies of RA patients, $n$ (\%) & $63(81.8 \%)$ \\
\hline \multicolumn{2}{|l|}{ DMARDs used during pregnancy } \\
\hline Patients without medication n (\%) & $23(29.87 \%)$ \\
\hline Patients with steroid monotherapy or csDMARDs and steroids $\mathrm{n}(\%)$ & $33(42.86 \%)$ \\
\hline Patients were on bDMARDs $\mathrm{n}(\%)$ & $21(27.27 \%)$ \\
\hline ESR before conception ${ }^{*}$, mean \pm SEM & $18.12 \pm 2.33^{*}$ \\
\hline CRP before conception ${ }^{*}$, mean \pm SEM & $11.28 \pm 1.90^{¥}$ \\
\hline
\end{tabular}

Note: ${ }^{¥}$ Valid $\mathrm{n}$ is given

Abbreviations: SEM, standard error of the mean; RA, rheumatoid arthritis; RF-CCP, rheumatoid factor-cyclic citrullinated peptide; ESR, erythrocyte sedimentation rate, CRP, C-reactive protein; DMARDs, disease modifying antirheumatic drugs. 
DMARDs used before conception were as follows: 48 (62.34\%) patients were on biologic bDMARDs with or without csDMARDs, 1 patient $(1.3 \%)$ was on tsDMARDs, tofacitinib and $28(36.36 \%)$ patients were using only csDMARDs. Of the patients using biologics, 19 were on etanercept, 11 on certolizumab, 8 on tocilizumab, 8 on adalimumab, and 1 patient each on infliximab and rituximab. Patients using only the csDMARDs, 5 were prescribed hydroxychloroquine (HCQ), 18 patients were given methotrexate, 9 patients were on sulfasalazine (SSZ), and 7 on concomitant use of $\leq 5 \mathrm{mg}$ steroids.

During pregnancy, 23 (29.87\%) patients were not on medication, 33 (42.86\%) patients were on steroid monotherapy or csDMARDs and steroids. Of the 16 patients who were on $\leq 10 \mathrm{mg}$ prednisolone, 5 were on prednisolone monotherapy and 11 on steroid in combination with either HCQ or SSZ. Twenty patients were on HCQ alone, 8 on SSZ alone, 21 (27.27\%) patients were on bDMARDs, of which 16 were on certolizumab, 4 on etanercept, and 1 patient was on tocilizumab.

Table 2 presents the comparison between the pregnant RA group and healthy pregnant controls. Our results showed that the age of conception, preterm labor, NICU admission, and low birth weight were statistically significant $(\mathrm{p}<0.05)$, and higher in pregnant RA patients than in healthy controls. No significant difference was observed in the events of the rate of abortion, stillbirths, congenital anomalies, cesarean section, ectopic pregnancy, and preeclampsia between pregnant RA patients and healthy controls $(\mathrm{p}>0.05)$.
The DAS28-CRP score of pregnant RA patients indicated that the disease remission occurred during preconception, within 3-6 months, 9 months of pregnancy and within 3 months after delivery in $67.53 \%, 71.42 \%, 73.33 \%$ and, $67.34 \%$ pregnant RA cases, respectively (Figure 1).

The relationship between the different clinical variables and RA pregnancy-related outcomes is illustrated in (Table 3 ), we observed that cases with longer disease duration ( $p$ $<0.001)$, high ESR $(\mathrm{p}<0.001)$, and high CRP levels ( $\mathrm{p}=$ 0.001 ) were statistically associated with cases of NICU admission; however, patients with higher age at conception $(p=0.003)$ were statistically related to stillbirth. Table 4 showed a significant relation between higher disease activity levels with abortion, preterm, and NICU admission pregnancy outcome $(p<0.05)$, however no statistically significant difference between the adverse pregnancy outcomes and csDMARDs, bDMARDs or prednisolone use ( $\mathrm{p} 0>0.05$ ).

The classification table obtained from the logistic model showed an overall accuracy of $76.1 \%$. Patients with mild and moderate-severe DAS28-CRP have significantly high chances of having an adverse pregnancy outcome (AOR $=11.44 ; 95 \%$ $\mathrm{CI}=1.598-81.90, \quad \mathrm{p}=0.015) \quad$ and $\quad(\mathrm{AOR}=100.30 ; \quad 95 \%$ $\mathrm{CI}=3.072-3275.90, \mathrm{p}=0.010)$ respectively with higher significance in moderate-severe disease activity (Table 5).

\section{Discussion}

RA influences pregnancy in different ways. It can affect patients' fertility as well as maternal and fetal outcomes. Before conception, it can affect patients' fertility, and after

Table 2 Comparison Between RA Group and Healthy Controls Groups

\begin{tabular}{|c|c|c|c|}
\hline Parameters & $\begin{array}{l}\text { Pregnancy Cases with RA } \\
\qquad(\mathrm{n}=\mathbf{7 7})\end{array}$ & $\begin{array}{l}\text { Pregnancy Cases as Healthy Controls } \\
\qquad(n=250)\end{array}$ & p-value \\
\hline Age at pregnancy, mean \pm SEM, years & $32.78 \pm 0.69^{¥}$ & $30.32 \pm 0.84$ & $0.034 *$ \\
\hline \multicolumn{4}{|l|}{ Neonatal outcomes } \\
\hline Newborn weight, mean \pm SEM, gm & $2701.50 \pm 87.39^{¥}$ & $3044.09 \pm 47.14$ & $0.001 *$ \\
\hline Congenital anomalies, $\mathrm{n}(\%)^{¥}$ & $\mathrm{I}(\mathrm{I} .3)$ & $4(1.6)$ & 0.849 \\
\hline Stillbirth, n (\%) ${ }^{¥}$ & $\mathrm{I}(1.3)$ & $3(1.2)$ & 0.944 \\
\hline Preterm birth, n (\%) & $17(22.1)$ & $21(8.4)$ & $0.001 *$ \\
\hline NICU admission, $\mathrm{n}(\%)^{*}$ & $3(3.9)$ & $0(0.0)$ & $0.001 *$ \\
\hline \multicolumn{4}{|l|}{ Pregnancy related } \\
\hline Preeclampsia, $n(\%)^{*}$ & $0(0.0)$ & I $(0.4)$ & 0.651 \\
\hline C-section, n (\%) & $8(10.39)$ & $17(6.8)$ & 0.298 \\
\hline Abortions, $\mathrm{n}(\%)^{¥}$ & $8(10.4)$ & $15(6.0)$ & 0.18 \\
\hline Ectopic pregnancy, n (\%) ${ }^{¥}$ & $0(0.0)$ & $2(0.8)$ & 0.429 \\
\hline
\end{tabular}

Notes: *Statistically significant at the $5 \%$ level of significance; ${ }^{*}$ valid $\mathrm{n}$ is given.

Abbreviations: SEM, standard error of the mean; RA, rheumatoid arthritis; NICU, neonatal intensive care unit; C-section, cesarean-section. 


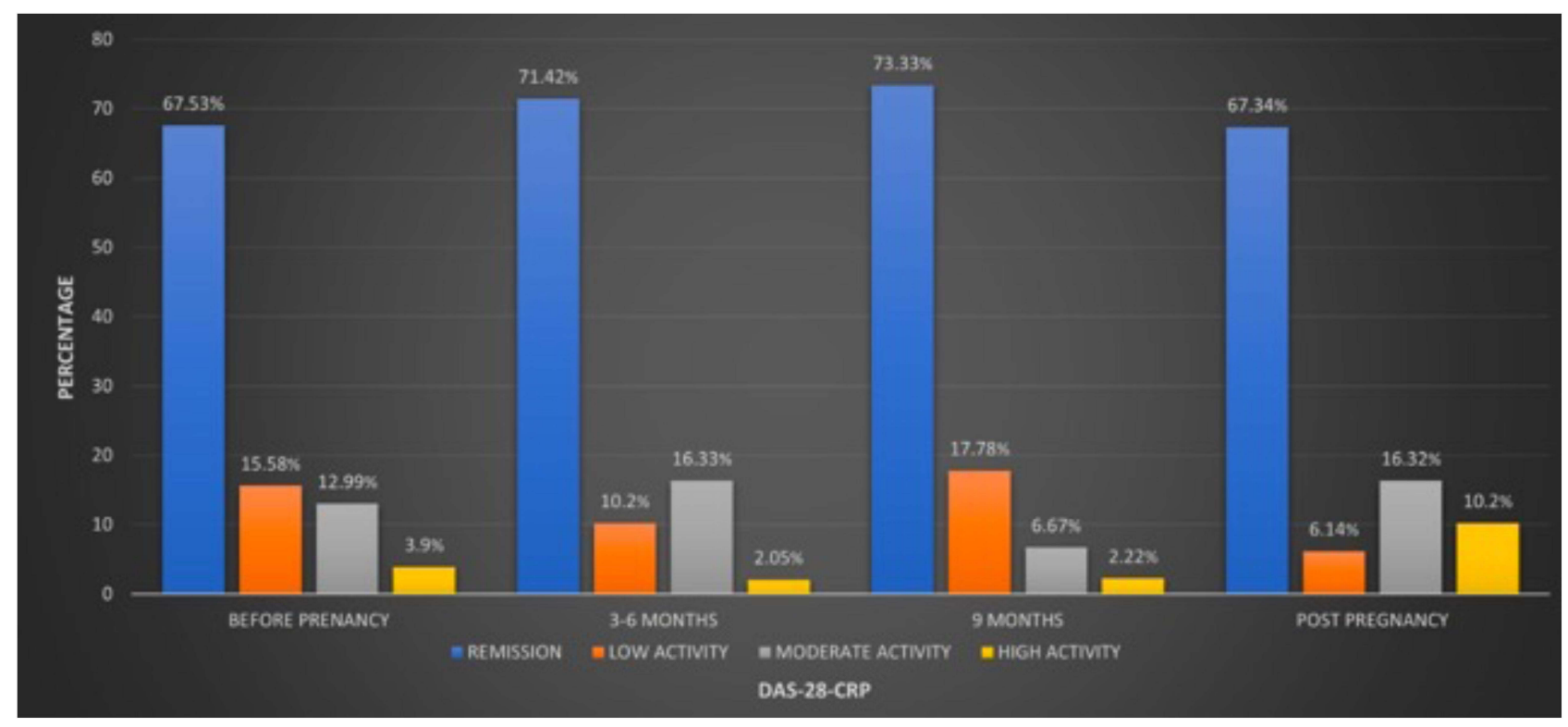

Figure I Comparison of DAS28-CRP before conception, during pregnancy (between 3-6 months and at 9 months), and post pregnancy.

conception, both maternal and fetal outcomes are at stake. ${ }^{1}$ We have studied a total of 77 pregnant patients with RA and 250 healthy age-matched pregnant controls. A total of $67.53 \%$ of RA patients were in remission before conception and $15.58 \%$ were having low disease activity score, and $81.8 \%$ of pregnancies were planned. Age of conception, preterm labor, neonatal intensive care unit (NICU) admission and low birth weight were higher in RA patients than healthy controls. Clinical and laboratory variables as longer disease duration and high $\mathrm{C}$-reactive protein and erythrocyte sedimentation rate levels before conception were associated with preterm NICU admission. The higher the disease activity the more frequent the adverse pregnancy outcome.
Most of the current studies either prospective pr $^{3,4}$ or retrospective $^{12,13}$ designed with limited data about the effect of planned follow-up protocol prospectively. To the best of our knowledge, the current study is considered the first prospectively designed pregnancy with a planned follow-up protocol in the Saudi population.

The mean age of patients with RA at conception was significantly higher than that of healthy controls, which is in agreement with a previous retrospective study by Nørgaard et al. ${ }^{5}$ This could be related to the high incidence of RA-related infertility or the need for disease control before conception. ${ }^{8}$

The majority $(80 \%)$ of our patients were in remission and had low disease activity, and $81.8 \%$ had a planned

Table 3 The Relation Between Clinical Variables and RA Pregnancy-Related Outcomes

\begin{tabular}{|c|c|c|c|c|c|c|}
\hline Parameters & $\begin{array}{c}\text { Total Adverse Pregnancy } \\
\text { Outcomes }(n=27)\end{array}$ & $\begin{array}{c}\text { Abortion } \\
(n=8)\end{array}$ & $\begin{array}{l}\text { Preterm } \\
(n=17)\end{array}$ & $\begin{array}{l}\text { Stillbirth } \\
(n=I)\end{array}$ & $\begin{array}{l}\text { NICU Admission (from } \\
\text { Preterm) }(n=3)\end{array}$ & p-value \\
\hline $\begin{array}{l}\text { Disease duration, mean } \\
\pm \mathrm{SEM}, \text { years }\end{array}$ & $6.07 \pm 0.68$ & $5.75 \pm 1.7$ & $6.41 \pm 0.76$ & $5.0 \pm 0.0$ & $9.67 \pm 0.88^{\dagger}$ & $<0.00 I^{*}$ \\
\hline $\begin{array}{l}\text { Age at pregnancy, mean } \\
\pm \text { SEM, years }\end{array}$ & $32.85 \pm 1.01$ & $34.25 \pm 2.3$ & $\begin{array}{c}32.53 \pm \\
1.10\end{array}$ & $25.0 \pm 0.0^{\dagger}$ & $31.67 \pm 5.23$ & $0.003 *$ \\
\hline ESR, mean \pm SEM & $27.74 \pm 3.62$ & $25.6 \pm 6.9$ & $\begin{array}{c}30.12 \pm \\
4.70\end{array}$ & $21.0 \pm 0.0$ & $53.67 \pm 2.33^{\dagger}$ & $<0.00 I^{*}$ \\
\hline CRP, mean \pm SEM & $|3.0 \pm 2.6|$ & $18.25 \pm 5.6$ & $\begin{array}{l}11.41 \pm \\
3.15\end{array}$ & $6.0 \pm 0.0$ & $24.78 \pm 14.32^{\dagger}$ & $0.001 *$ \\
\hline
\end{tabular}

Notes: *Statistically significant at the $5 \%$ level of significance; ${ }^{\dagger}$ significant difference with others.

Abbreviations: SEM, standard error of the mean; RA, rheumatoid arthritis; NICU, neonatal intensive care unit; ESR, erythrocyte sedimentation rate, CRP, C-reactive protein. 
Table 4 Association Between Smoking, Medications Used During Pregnancy and Disease Activity Scoring at Conception and RA Pregnancy-Related Outcomes

\begin{tabular}{|c|c|c|c|c|c|c|c|c|}
\hline \multirow[t]{3}{*}{ Clinical Parameters } & \multicolumn{8}{|c|}{ Adverse Pregnancy Outcome } \\
\hline & \multicolumn{2}{|c|}{$\begin{array}{l}\text { Preterm } \\
\begin{array}{l}\text { N=I7 } \\
\text { n (\%) }\end{array}\end{array}$} & \multicolumn{2}{|c|}{$\begin{array}{l}\text { NICU Admission (from Preterm) } \\
\qquad \begin{array}{c}\mathrm{N}=3 \\
\mathrm{n}(\%)\end{array}\end{array}$} & \multicolumn{2}{|c|}{$\begin{array}{c}\text { Abortion } \\
\begin{array}{c}\mathrm{N}=8 \\
\mathrm{n}(\%)\end{array}\end{array}$} & \multicolumn{2}{|c|}{$\begin{array}{l}\text { Stillbirth } \\
\qquad \begin{array}{l}\mathbf{N}=\mathbf{I} \\
\mathrm{n}(\%)\end{array}\end{array}$} \\
\hline & No & Yes & No & Yes & No & Yes & No & Yes \\
\hline \multicolumn{9}{|l|}{ Smoker } \\
\hline No & $47(75.8)$ & $15(24.2)$ & $60(96.8)$ & $2(3.20)$ & $55(88.7)$ & $7(\mid 1.3)$ & $61(98.4)$ & I $(1.60)$ \\
\hline Yes & $3(60.0)$ & $2(40.0)$ & $4(80.0)$ & I (20.0) & $4(80.0)$ & I (20.0) & $5(100.0)$ & $0(0.00)$ \\
\hline$p$-value & \multicolumn{2}{|c|}{0.595} & \multicolumn{2}{|c|}{0.211} & \multicolumn{2}{|c|}{0.482} & \multicolumn{2}{|c|}{0.775} \\
\hline \multicolumn{9}{|l|}{ bDMARDs } \\
\hline No & $30(69.8)$ & $13(30.2)$ & $4 \mathrm{I}(95.3)$ & $2(4.70)$ & $38(88.4)$ & $5(11.6)$ & $42(97.7)$ & I (2.3) \\
\hline Yes & $20(83.3)$ & $4(16.70)$ & $23(95.8)$ & I (4.20) & $21(87.5)$ & $3(12.5)$ & $24(100)$ & $0(0.00)$ \\
\hline$p$-value & \multicolumn{2}{|c|}{0.221} & \multicolumn{2}{|c|}{0.927} & \multicolumn{2}{|c|}{0.916} & \multicolumn{2}{|c|}{0.452} \\
\hline \multicolumn{9}{|l|}{ Prednisolone } \\
\hline No & $43(78.2)$ & $12(21.8)$ & $54(98.2)$ & I (I.80) & $48(87.3)$ & $7(12.7)$ & $54(98.2)$ & I (I.8) \\
\hline Yes & $7(58.3)$ & $5(4 \mid .7)$ & $10(83.3)$ & $2(16.7)$ & II (9I.7) & I (8.3) & $12(100)$ & $0(0.00)$ \\
\hline$p$-value & \multicolumn{2}{|c|}{0.164} & \multicolumn{2}{|c|}{0.080} & \multicolumn{2}{|c|}{0.671} & \multicolumn{2}{|c|}{0.638} \\
\hline \multicolumn{9}{|l|}{ csDMARDs } \\
\hline No & $29(69.0)$ & $13(31.0)$ & $4 I(97.6)$ & I (2.40) & 39 (92.9) & $3(7.1)$ & $4 I(97.6)$ & I (2.4) \\
\hline Yes & $21(84.0)$ & $4(16.0)$ & $23(92.0)$ & $2(8.00)$ & $20(80.0)$ & $5(20.0)$ & $25(100)$ & $0(0.00)$ \\
\hline$p$-value & \multicolumn{2}{|c|}{0.174} & \multicolumn{2}{|c|}{0.551} & \multicolumn{2}{|c|}{0.138} & \multicolumn{2}{|c|}{0.437} \\
\hline \multicolumn{9}{|l|}{ DAS28-CRP } \\
\hline Remission & $35(83.3)$ & $7(16.7)$ & $42(100)$ & $0(0.0)$ & $40(95.2)$ & $2(4.80)$ & $42(100)$ & $0(0.0)$ \\
\hline Mild & $10(83.3)$ & $2(16.7)$ & $12(100)$ & $0(0.0)$ & $9(75.0)$ & $3(25.0)$ & II (9I.7) & I (8.3) \\
\hline Moderate-Severe & $5(38.5)$ & $8(6 \mid .5)$ & $10(76.9)$ & $3(23.1)$ & $10(76.9)$ & $3(23.1)$ & $13(100)$ & $0(0.0)$ \\
\hline$p$-value & \multicolumn{2}{|c|}{$0.006^{*}$} & \multicolumn{2}{|c|}{$0.011 *$} & \multicolumn{2}{|c|}{$0.030 *$} & \multicolumn{2}{|c|}{0.179} \\
\hline
\end{tabular}

Note: *Statistically significant.

pregnancy. This figure was higher than those of another study $^{12}$ in which $75 \%$ were planned pregnancy and $54 \%$ had mild disease activity scores before conception. Nevertheless, our study was prospective and $62.34 \%$ of our patients were on biologics of variable classes. Approximately $30 \%$ were off-medication during pregnancy, the number being lower than that reported by Eudy et $a 1,{ }^{12}$ in which half of the patients were offmedications during pregnancy.

We have reported a comparable abortion rate between planned pregnancy RA patients vs healthy controls. Brouwer et $\mathrm{al}^{14}$ reported that the risk of abortion in women with RA is comparable to that in the general population. Most of our cohort consisted of RA patients with a planned pregnancy, implicating that disease remission can be associated with better outcomes. Surprisingly, we found it even better than the general population with the usual unplanned pregnancy. The retrospective study by Eudy et $\mathrm{al}^{12}$ revealed a better outcome in the planned RA pregnancy; all the patients had early deliveries and episodes of preeclampsia; only $50 \%$ were taking RA-related medications, although the study design of being prospective in our cohort and different ethnicity could be the reason behind the difference. ${ }^{12}$

Norgaard et $\mathrm{al}^{5}$ have reported a slight increase in the risk of preeclampsia from $3.4 \%$ in control to $5.0 \%$ in RA patients, while other studies did not confirm these findings, probably due to lack of power. ${ }^{4}$ Two prior retrospective 
Table 5 Binary Logistic Regression Using Backward Selection for Assessing the Relation Between Clinical Variables and RA PregnancyRelated Outcomes

\begin{tabular}{|l|c|c|c|c|c|c|c|}
\hline Parameters & $\beta$ & S.E. & Wald & p-value & $\begin{array}{c}\text { Adjusted } \\
\text { Odds Ratio }\end{array}$ & \multicolumn{2}{|c|}{$95 \%$ C.I. } \\
\hline Age at pregnancy & 0.538 & 0.604 & 0.795 & 0.373 & 1.713 & 0.525 & 5.59 \\
Age of diagnosis & -0.447 & 0.606 & 0.543 & 0.461 & 0.64 & 0.195 & 2.098 \\
Disease Duration & -0.529 & 0.635 & 0.695 & 0.404 & 0.589 & 0.17 & 2.045 \\
Seropositive & -0.393 & 0.814 & 0.234 & 0.629 & 0.675 & 0.137 & 3.324 \\
Smoker & -0.129 & 1.794 & 0.005 & 0.943 & 0.879 & 0.026 & 29.586 \\
ESR & -0.017 & 0.04 & 0.183 & 0.669 & 0.983 & 0.909 & 1.063 \\
CRP & 0.023 & 0.043 & 0.289 & 0.591 & 1.023 & 0.941 & 1.114 \\
bDMARDs & -1.705 & 0.916 & 3.463 & 0.063 & 0.182 & 0.03 & 1.095 \\
Prednisolone & -1.41 & 1.183 & 1.422 & 0.233 & 0.244 & 0.024 & 2.478 \\
csDMARDs & -1.176 & 0.9 & 1.706 & 0.191 & 0.309 & 0.053 & 1.801 \\
DAS28-CRP (Mild) & 2.437 & 1.004 & 5.888 & $0.015 *$ & 11.44 & 1.598 & 81.903 \\
DAS28-CRP (Moderate-Severe) & 4.608 & 1.779 & 6.713 & $0.001 *$ & 100.308 & 3.072 & 3275.802 \\
\hline
\end{tabular}

Note: *Statistically significant.

studies ${ }^{5,15}$ have claimed that there is a modestly increased risk for preterm birth and preeclampsia in pregnant women with RA, although absolute numbers reported were relatively low. Some reports have shown an increased risk for cesarean delivery (26-34\%) compared with (16.5-19.5\%) in the general population. ${ }^{5,15}$ Active disease was associated with an increased risk for cesarean section in $22 \%,{ }^{15}$ however, most of our patients were in remission with low disease activity; further, cesarean deliveries vary with different countries.

There was no increased risk of fetal-related congenital malformations or perinatal death for any pregnant women with RA; these findings were similar to those reported in previous studies. ${ }^{15,16}$

The current study revealed that preterm labor, NICU admission, and low fetal birth weight were higher in pregnant patients with RA than in healthy controls, which is in agreement with previous studies. ${ }^{5,12,15-17}$ Moreover, our analyses showed that longer disease duration, and higher CRP and ESR levels before conception were associated with preterm NICU admission, however, higher age at disease onset was associated with abortion, and stillbirth was associated with higher age at conception. Higher disease activity was associated with abortion, preterm, and NICU admission pregnancy outcome. Binary logistic analysis showed that the higher the disease activity the higher the risk of the adverse pregnancy outcomes. Alike our results de Man et $\mathrm{al}^{4}$ reported that an increase in the lower birth weight of infants for patients with RA was related to the disease activity during pregnancy. Cytokine concentrations like high IL-6 and low IL-10 levels were found to be associated with low fetal birth weight and poorer pregnancy outcomes in pregnant women with RA, apart from teratogenic medications and high disease activity. $^{18}$

A strong risk of preterm delivery associated with high disease activity was also reported. ${ }^{19}$ The same study also indicated an association of preterm delivery with comorbid autoimmune disease, use of $>10 \mathrm{mg}$ prednisone, previous history of preterm labor, and previous intrauterine growth restriction. In our study, there was no association between prednisolone use and adverse outcome, as most of the RA patients were in remission and using $<10 \mathrm{mg}$ prednisolone. Besides, we had excluded patients having comorbid autoimmune diseases.

This study had some limitations. The small sample size of pregnant cases, to assess the exact percentage of planned pregnancy in healthy controls; however, planned pregnancy protocols are usually applied and recommended in women with chronic diseases, rather than in the general population. Another limitation where RA participants were older, which could interact with all adverse outcomes, a small population of pregnant women with active RA; however, most of our patients had planned pregnancy protocol, in which the recommendation advice is to conceive in low disease activity or remission stage for better fertility and outcome. ${ }^{20,21}$ 


\section{Conclusion}

We conclude that RA is associated with numerous adverse pregnancy-related outcomes. Patients with active disease were at a higher risk for adverse pregnancy outcomes, including a high risk of preterm labor with or without NICU admission and abortion; nevertheless, tight disease control should be aimed. Disease remission before conception is advised. Pregnant women with RA should be educated and encouraged to plan their pregnancies with their physician as other patients with chronic diseases.

\section{Abbreviations}

RA, rheumatoid arthritis; DAS 28, disease activity scoring; NICU, neonatal intensive care unit; CRP, C-reactive protein; ESR, erythrocyte sedimentation rate; ACR, American College of Rheumatology; EULAR, European League Against Rheumatism; RF, rheumatoid factor; Anti-CCP Abs, anticyclic citrullinated peptide antibodies; CMIA, chemiluminescence microparticle immunoassay; ESR, erythrocyte sedimentation rate; $\mathrm{CRP}, \mathrm{C}$ reactive protein; ELISA, enzyme-linked immunosorbent assay; csDMARDs, conventional synthetic disease modifying antirheumatic drugs; bDMARDs, biological disease modifying antirheumatic drugs; tsDMARDs, targeted synthetic disease modifying antirheumatic drugs.

\section{Funding}

This research received no specific grant from any funding agency in the public, commercial, or not-for-profit sectors.

\section{Disclosure}

Dr Mufaddal Adil Alaithan reports personal fees from Pfizer, outside the submitted work. The authors declare no other conflicts of interest.

\section{References}

1. de Jong PHP, Dolhain RJ. Fertility, pregnancy, and lactation in rheumatoid arthritis. Rheum Dis Clin North Am. 2017;43(2):227-237.

2. Chakravarty EF. Rheumatoid arthritis and pregnancy: beyond smaller and preterm babies. Arthritis Rheum. 2011;63:1469-1471. doi:10.1002/ art.30206

3. de Man YA, Dolhain RJ, van de Geijn FE, et al. Disease activity of rheumatoid arthritis during pregnancy: results From a Nationwide Prospective Study. Arthritis Rheum. 2008;59:1241-1248. doi:10.1002/ art.24003

4. de Man YA, Hazes JM, van der Heide H, et al. Association of higher rheumatoid arthritis disease activity during pregnancy with lower birth weight: results of a National Prospective Study. Arthritis Rheum. 2009;60:3196-3206. doi:10.1002/art.24914
5. Nørgaard M, Larsson H, Pedersen L, et al. Rheumatoid arthritis and birth outcomes: a Danish and Swedish nationwide prevalence study. J Intern Med. 2010;268:329-337. doi:10.1111/j.1365-2796.2010.02239.x

6. de Steenwinkel FD, Hokken-Koelega AC, de Ridder MA, et al. Rheumatoid arthritis during pregnancy and postnatal catch-up growth in the off-spring. Arthritis Rheum. 2014;66:1705-1711. doi:10.1002/ art.38519

7. Verma A, Shrimali L. Maternal body mass index and pregnancy outcome. J Clin Diagn Res. 2012;6:1531-1533. doi:10.7860/JCDR/ 2012/4508.2551

8. Aletaha T, Neogi AJ, Silman J, et al. 2010 Rheumatoid arthritis classification criteria: an American College of Rheumatology/ European League Against Rheumatism collaborative initiative. Arthritis Rheum. 2010;62:2569-2581. doi:10.1002/art.27584

9. Blencowe H, Cousens S, Oestergaard M, et al. National, regional, and worldwide estimates of preterm birth rates in the year 2010 with time trends since 1990 for selected countries: a systematic analysis and implications. Lancet. 2012;379(9832):2162-2172. Estimates from 2010. doi:10.1016/S0140-6736(12)60820-4

10. American College of Obstetricians and Gynecologists. Perinatal care at the threshold of viability. ACOG Practice Bulletin \#38. Washington DC: American College of Obstetricians and Gynecologists; 2002.

11. Aletaha D, Nell VP, Stamm T, et al. Acute phase reactants add little to composite disease activity indices for rheumatoid arthritis: validation of a clinical activity score. Arthritis Res Ther. 2005:7R796-806.

12. Eudy AM, McDaniel G, Clowse MEB. Pregnancy in rheumatoid arthritis: a retrospective study. Clin Rheumatol. 2018;37:789-794. doi:10.1007/s10067-017-3939-4

13. Lin HC, Chen SF, Lin HC, Chen YH. Increased risk of adverse pregnancy outcomes in women with rheumatoid arthritis: a nationwide population-based study. Ann Rheum Dis. 2010;69:715-717. doi:10.1136/ard.2008.105262

14. Brouwer J, Laven JS, Hazes JM, et al. Brief report: abortion in female rheumatoid arthritis patients: associations with serologic findings, disease activity, and antirheumatic drug treatment. Arthritis Rheuml. 2015;67:1738-1743. doi:10.1002/art.39137

15. Reed SD, Vollan TA, Svec MA. Pregnancy outcomes in women with rheumatoid arthritis in Washington State. Matern Child Health J. 2006;10:361-366. doi:10.1007/s10995-006-0073-3

16. Wolfberg AJ, Lee-Parritz A, Peller AJ, et al. Association of rheumatologic disease with preeclampsia. Obstet Gynecol. 2004;103:1190-1193. doi:10.1097/01.AOG.0000126279.87151.e1

17. Rom AL, Wu CS, Olsen J, et al. Fetal growth and preterm birth in children exposed to maternal or paternal rheumatoid arthritis: a nationwide cohort study. Arthritis Rheumatol. 2014;66(12):3265-73. doi:10.1002/art.38874.

18. Atta DS, Girbash EF, Abdelwahab SM, et al. Maternal cytokines and disease severity influence pregnancy outcomes in women with rheumatoid arthritis. J Matern Fetal Neonatal Med. 2016;29:3358-3363. doi:10.3109/14767058.2015.1127342

19. Bharti B, Lee SJ, Lindsay SP, et al. Disease severity and pregnancy outcomes in women with rheumatoid arthritis: results from the Organization of Teratology Information Specialists Autoimmune Diseases in Pregnancy Project. $J$ Rheumatol. 2015;42:1376-1382. doi:10.3899/jrheum.140583

20. Krause ML, Makol A. Management of rheumatoid arthritis during pregnancy: challenges and solutions. Open Access Rheumatol. 2016;8:23-36. doi:10.2147/OARRR.S85340

21. De Cock D, Brants L, Soenen I, et al. A systematic review on the effect of DMARDs on fertility in rheumatoid arthritis. Semin Arthritis Rheum. 2020;50(5):873-878. PMID: 32896703. doi:10.1016/j. semarthrit.2020.07.003 


\section{Publish your work in this journal}

Open Access Rheumatology Research and Reviews is an international, peer-reviewed, open access journal publishing original research, reports, editorials, reviews and commentaries on all aspects of clinical and experimental rheumatology in the clinic and laboratory including the following topics: Pathology, pathophysiology of rheumatological diseases; Investigation, treatment and management of rheumatological diseases; Clinical trials and novel pharmacological approaches for the treatment of rheumatological disorders. The manuscript management system is completely online and includes a very quick and fair peer-review system, which is all easy to use. Visit http://www.dovepress.com/testimonials.php to read real quotes from published authors. 